\title{
Fabrication of multiperiod $\mathrm{Si} / \mathrm{SiO}_{2} / \mathrm{Ge}$ layered structure through chemical bond manipulation
}

\author{
K. Prabhakaran, ${ }^{\text {a) }}$ T. Matsumoto, ${ }^{\text {b) }}$ T. Ogino, and Y. Masumoto ${ }^{\text {b) }}$ \\ NTT Basic Research Laboratories, 3-1 Morinosato Wakamiya, Atsugi-Shi, Kanagawa-ken 243-0198 Japan
}

(Received 18 November 1997; accepted for publication 13 April 1998)

\begin{abstract}
In this letter, we report a method called chemical bond manipulation for fabrication of multiperiod nanometer sized $\mathrm{Si} / \mathrm{SiO}_{2} / \mathrm{Ge}$ layered structure. Chemical bond manipulation is a self-organization process which involves selective breaking and making of surface chemical bonds and thereby enable formation of the desired species on a full wafer scale. We show that oxygen of germanium oxide layer formed on $\mathrm{Si}(111)$ are picked up by the $\mathrm{Si}$ atoms arriving at the surface during subsequent growth. This phenomenon involves breaking of $\mathrm{Ge}-\mathrm{O}$ bonds and making of $\mathrm{Si}-\mathrm{O}$ bonds and leads to the formation of ultrathin $\mathrm{Si}$ and Ge layers sandwiched between ultrathin silicon oxide layers, preserving the original wafer morphology. This material exhibits blue-green light emission at room temperature when excited by ultraviolet laser. (C) 1998 American Institute of Physics.

[S0003-6951(98)01324-2]
\end{abstract}

Light emission from group IV semiconductors has been emerging as one of the important research fields, aimed at developing Si-based optoeletronics technologies. ${ }^{1-4} \mathrm{Si}$ has been reported to emit light when it is in nanostructure form ${ }^{5,6}$ and the luminescence behavior is crucially dependent on the nature of the nanometer sized structures. However, controllability of the size of the nanostructures, interfaces, and surface planarity have posed problems in realizing integrated $\mathrm{Si}$ optoelectronic devices. ${ }^{6}$ In the area of fabrication of nanostructured materials, control of atomic processes is a key approach to achieving the formation of the desired species. Several researchers have reported ${ }^{7}$ nanostructure fabrication by atom manipulation such as using scanning tunneling microscope tip. However, this approach has the main drawback that it is limited to only a very small area of the wafer and involve only a single type of material and therefore is not technologically feasible. Chemical bond manipulation involves a judicious combination of deposition techniques such as molecular beam epitaxy (MBE) and surface phenomena such as chemical reaction. Through manipulation of surface chemical bonds, ${ }^{8}$ it is possible to impart functionality to nanostructures by forming different phases of materials such as semiconductor, metal, and insulators which are essential for devices.

The growth of $\mathrm{Si}$ and $\mathrm{Ge}$ was carried out in an MBE chamber. In situ ultraviolet and X-ray photoelectron spectroscopic measurements (UPS and XPS) were performed to characterize the surface species and the reaction pathways. $\operatorname{Si}(111)$ wafers ( $n$ type, $1-5 \Omega \mathrm{cm}$ ) were cleaned using standard methods and a buffer layer of $\sim 150 \AA$ was deposited to ensure good quality starting surface. On this sample, 4 monolayers $(\sim 6.5 \AA)$ of Ge was deposited at room temperature (RT). The sample was oxidized by exposing to flowing oxygen, outside the ultrahigh vacuum (UHV) chamber, for 1 min. This process resulted in the formation of nearly two monolayers of $\mathrm{Ge}$ oxide. The sample was reinserted into the UHV chamber immediately and characterized using photo-

\footnotetext{
${ }^{\text {a)} E l e c t r o n i c ~ m a i l: ~ p r a b @ w i l l . b r l . n t t . c o . j p ~}$

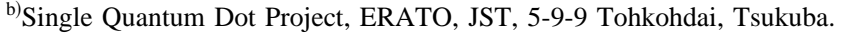

emission measurements. Si $(3 \mathrm{~nm})$ was deposited onto this sample at RT. This procedure (Ge deposition, oxidation, and $\mathrm{Si}$ deposition) was repeated seven times (in the case of the sample discussed in this letter, hereafter called as 7-period sample), each time characterizing the surface species. Finally, the sample was capped with Ge $(6.5 \AA)$ and Si $(5 \mathrm{~nm})$ and ex situ measurements such as cross-sectional transmission electron microscopy (XTEM), Auger depth profiling, atomic force microscopy (AFM), and photoluminescence (PL) measurements were performed. We preferred $\mathrm{Si}(111)$ substrate to $\mathrm{Si}(100)$ for the growth of multilayers because our previous work ${ }^{9}$ showed that bonding partner change reaction performed on $\mathrm{Si}(100)$ substrate resulted in considerable surface roughening.

Fig. 1 shows the XPS in the Ge $2 p$ region after oxidizing the Ge covered $\mathrm{Si}(111)$ surface [spectrum (a)], and after depositing Si onto the Ge oxide covered surface [spectrum (b)]. Spectrum (a) shows that oxidation results in the formation of a mixture of $\mathrm{Ge}$ oxides. On depositing $\mathrm{Si}$ onto this surface, $\mathrm{Ge}-\mathrm{O}$ bonds are broken and $\mathrm{Si}-\mathrm{O}$ bonds are formed. In other words, oxygen changes the bonding partner from $\mathrm{Ge}$ to $\mathrm{Si}^{10}$ and the signal due to the oxides in Ge $2 p$ spectrum disappears completely. The Ge $2 p$ spectrum returns to that of the same prior to oxidation, implying complete reduction [see spectrum (b) in Fig. 1]. Corresponding Si $2 p$ spectra are plotted in the inset to Fig. 1 and show signal due to silicon oxides after the deposition of $\mathrm{Si}$ onto the Ge oxide covered $\mathrm{Si}(111)$ surface. The signal due to the oxides in $\mathrm{Si} 2 p$ is weak compared to that of the Ge oxides because the main peak is dominated by bulk Si. Spectral changes in $\mathrm{O} 1 s$ as well as UPS corroborate the chemical bond manipulation reaction. This breaking of $\mathrm{Ge}-\mathrm{O}$ bonds and making of $\mathrm{Si}-\mathrm{O}$ bonds can be understood based on the large differences in the heats of formation ${ }^{11}$ of $\mathrm{Ge}$ oxides (for $\mathrm{GeO}$ and $\mathrm{GeO}_{2}$, $\Delta H_{f}=-62$ and $-131 \mathrm{kcal} / \mathrm{mol}$, respectively) compared to that of Si oxide $\left(\Delta H_{f}=-217 \mathrm{kcal} / \mathrm{mol}\right)$. The strength of the chemical bonds are accordingly different ( $\mathrm{Ge}-\mathrm{O}$ and $\mathrm{Si}-\mathrm{O}$, 151 and $191 \mathrm{kcal} / \mathrm{mol}$, respectively). The O $1 \mathrm{~s}$ core level exhibits a peak around 531.2 eV from oxidized Ge layer and after depositing $\mathrm{Si}$, the peak shifts to $532.4 \mathrm{eV}$ due to the 


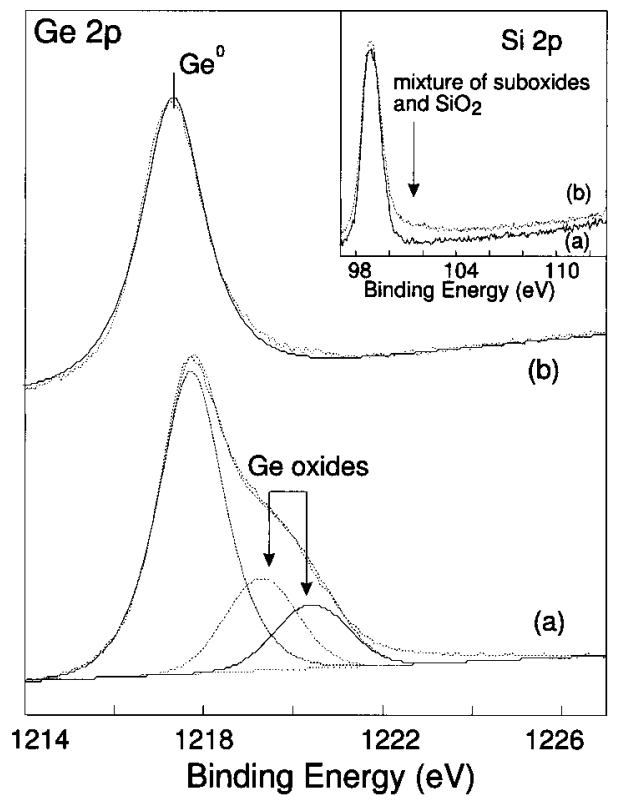

FIG. 1. XPS in the Ge $2 p$ region from a thin (4 ML) Ge layer deposited on $\mathrm{Si}(111)$ and oxidized [spectrum (a)]. The component peaks due to germanium oxides are resolved. Spectrum (b) is obtained after depositing $20 \AA \mathrm{Si}$ onto this sample at RT, indicating the disappearance of germanium oxide peaks. The height has been normalized to make the comparison clearer. Inset shows the corresponding $\operatorname{Si} 2 p$ spectra. Signal due to silicon oxide formation on depositing $\mathrm{Si}$ onto the germanium oxide covered surface is indicated.

formation of $\mathrm{Si}-\mathrm{O}$ bonds. This was confirmed after comparing with independent oxidation experiments on clear $\mathrm{Si}$ and Ge wafers. Changes in the He I UPS further substantiates the occurrence of the reaction. After oxidizing the Ge capped $\mathrm{Si}(111)$ and the main signal due to $\mathrm{Ge}-\mathrm{O}$ bonds is observed at $5.6 \mathrm{eV}$. Upon depositing $\mathrm{Si}$ onto this surface, the main peak is observed at a remarkably different binding energy of $6.6 \mathrm{eV}$ due to the formation of $\mathrm{Si}-\mathrm{O}$ bonds. Furthermore, cross-sectional TEM pictures as well as AFM images indicate that $\mathrm{Si}$ atoms reaching the surface in excess of the reaction with the Ge oxides, form a uniform layer on top of the silicon oxide. By repeating the above procedure, a novel structure consisting of ultrathin $\mathrm{Si}$ and Ge layers sandwiched between ultrathin silicon oxide layers, was fabricated (shown schematically in Fig. 2).

Figure 2 is the XTEM image from the 7-period sample, (a)

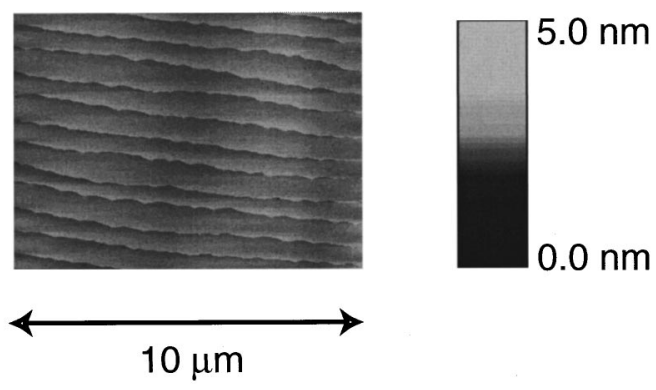

(b)

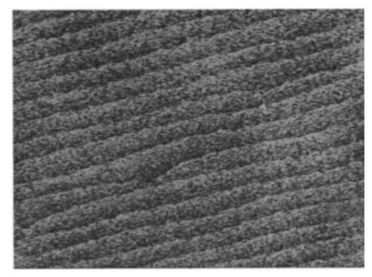

FIG. 3. Surface morphology (AFM) of (a) clean $\mathrm{Si}(111)$ substrate and (b) 7-period sample. The original morphology is preserved, as indicated by the clear observation of surface steps.

indicating the formation of continuous layers with an interface roughness of the order of atomic dimensions. In Fig. 2 we also show the Auger depth profiling data from this sample. The sharp dips observed in the Si $L V V$ signal due to the depletion of elemental $\mathrm{Si}$ and the corresponding peaks in O KLL signal (as indicated by the arrows), show the formation of silicon oxide layers after each deposition of $\mathrm{Si}$ atoms. Figure 3 shows the morphology of the clean substrate and the 7-period sample, as observed by AFM [images (a) and (b), respectively]. In spite of the overlayer growth (total thickness $\sim 30 \mathrm{~nm}$ ) and the occurrence of chemical bond manipulation in each period, the final surface of the sample clearly shows the step/terrace structure, similar to that of the starting surface. In other words, the original wafer morphology is preserved throughout the fabrication process. This is mainly because, the chemical bond manipulation is performed at RT, where the atom diffusion rates responsible for interface roughening are considerably reduced. ${ }^{12}$ This helps to maintain a smooth interface, reduce the strain in each layer, and thereby maintain surface planarity. This is a

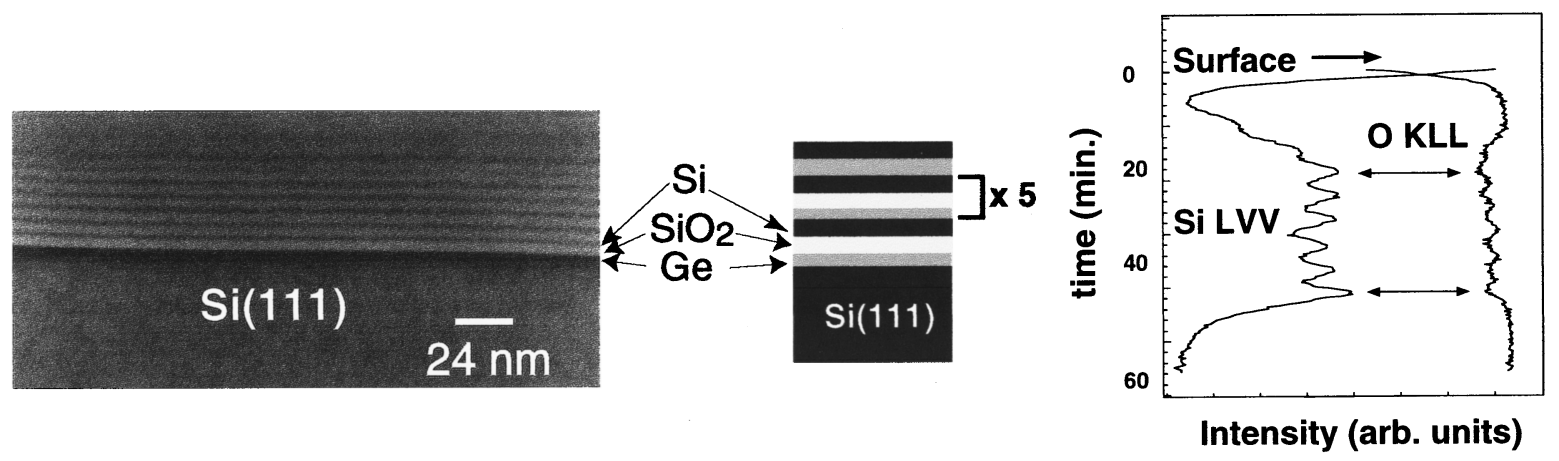

FIG. 2. XTEM image from the 7-period sample showing the formation of continuous layers and sharp interfaces. A schematic description of the sample is also given. Auger depth profile data from the 7-period sample is also shown. The sharp dips in the $\mathrm{Si} L V V$ signal (due to depletion of elemental $\mathrm{Si}$ ) and the corresponding peaks in O KLL signal (as indicated by the arrows), confirm the formation of silicon oxide layers, as a result of the chemical bond manipulation. 


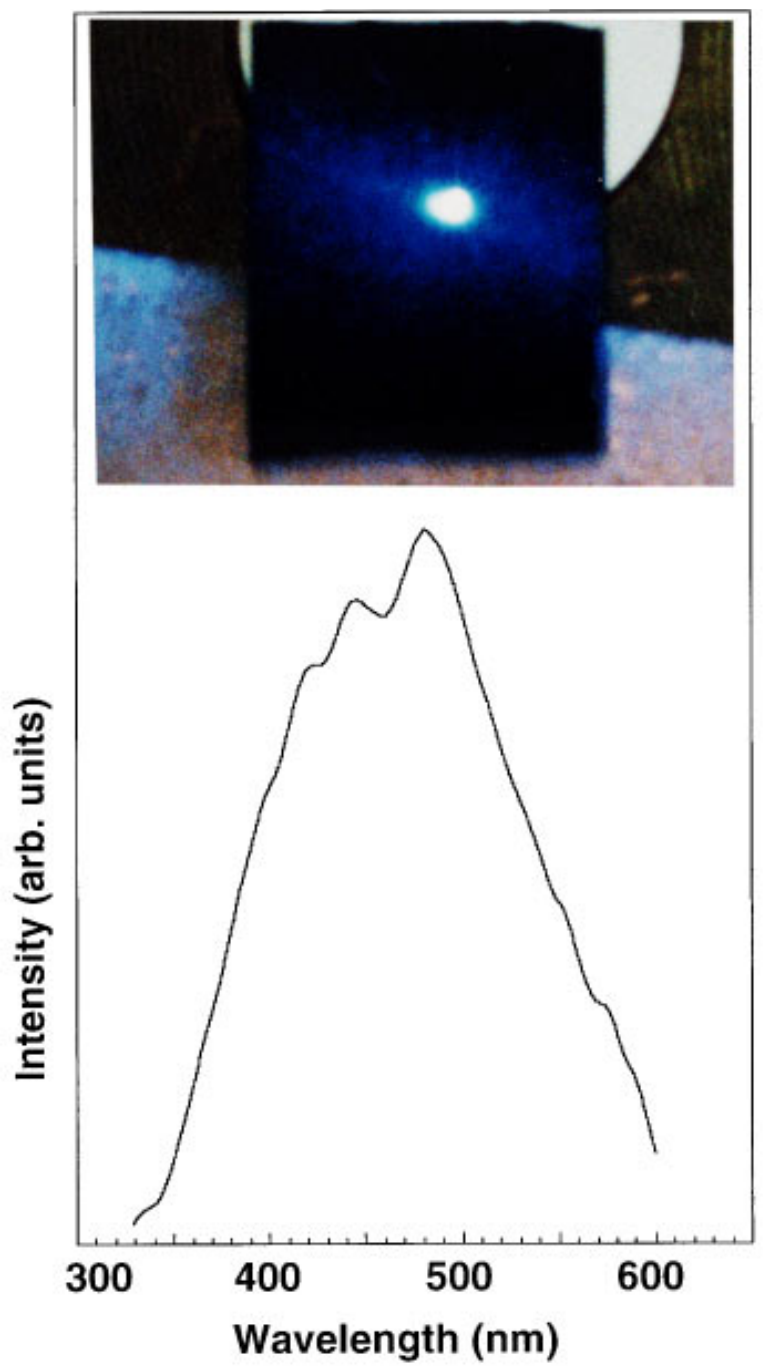

FIG. 4. RT PL spectrum from the 7-period sample excited by He-Cd laser $(325 \mathrm{~nm}, \sim 2 \mathrm{~mW}$ ). Inset shows the photograph of the emission (beam spot size $\sim 0.5 \mathrm{~mm}$ ) taken at a sample temperature of $13 \mathrm{~K}$.

unique aspect of the chemical bond manipulation and permits the formation of the new layered structure on a full wafer scale. It is to be noted that on annealing at $500{ }^{\circ} \mathrm{C}$, the layer structure is destroyed and the sample surface becomes rough, as the AFM images show the presence of nanometer sized voids.

Figure 4 shows the PL spectrum measured using $325 \mathrm{~nm}$ excitation light from a He-Cd laser. Blue-green luminescence is observed at RT with a spectral peak at $\sim 2.6 \mathrm{eV}$ (full width at half maximum of the order of $1 \mathrm{eV}$ ). The inset to Fig. 4 is a photograph of the light emitting sample at a temperature of $13 \mathrm{~K}$. We recorded the PL intensity as a function of temperature and found that the intensity decreases when the sample is brought to room temperature from $13 \mathrm{~K}$. Additionally, we fabricated samples with different periods (4 and 2) and found that the PL intensity from those samples were significantly low compared to that of the 7-period sample.

We fabricated the multilayer structured samples with varying thicknesses of $\mathrm{Si}$ and $\mathrm{Ge}$ and performed PL measurements, in order to examine the effect of quantum confinement on the blue-green light emission. We did not observe Downloaded 21 May 2004 to 130.158.105.241. Redistribution subject any shift in the PL peak energy and therefore can rule out the quantum confinement effect as a possible explanation for the light emission. We can also conclude that the emission is not due to nanocrystals/nanoparticles of $\mathrm{Si}^{13}$ or $\mathrm{Ge}^{14}$ as the samples do not contain them, as verified by XTEM (the entire fabrication process is carried out at room temperature). We can also exclude the possibility that the emission originates from Ge oxides ${ }^{15}$ because XPS results clearly show that $\mathrm{Ge}$ is completely in the elemental form (see Ge $2 p$ spectra in Fig. 1). Other possibility for the light emission is defects created at the interface or in the silicon oxide layer. ${ }^{16-18}$ It is known that defects in $\mathrm{SiO}_{2}$ matrix and nonstoichiometric oxide of $\mathrm{Si}\left(\mathrm{SiO}_{x}\right.$ where $\left.x<2\right)$ can luminesce efficiently ${ }^{19,20}$ and the emission observed at $2.7 \mathrm{eV}$ is assigned as originating from oxygen vacancy. ${ }^{21}$ In our samples, the signal due to oxides in the $\mathrm{Si} 2 p$ spectrum after the occurrence of the reaction, is a broad feature and therefore indicate the presence of a mixture of suboxides and $\mathrm{SiO}_{2}$ (indicated by the arrow in the inset to Fig. 1). This may suggest that the emission is suboxide related. Additionally, it is possible that in our samples isolated luminescent species, such as for example, $E^{\prime}$ center are created as a result of the bonding partner change reaction. However, such species, if at all present in our samples, are below the detection level in techniques such as electron spin resonance spectroscopic measurements. Tamura et $_{\text {al. }}{ }^{22}$ reported that hydroxyl groups $(-\mathrm{OH})$ present in the system can cause blue-green luminescence and that is a possibility which cannot be ruled out in our samples.

${ }^{1}$ S. S. Iyer and Y. H. Xie, Science 260, 40 (1993).

${ }^{2}$ Z. H. Lu, D. J. Lockwood, and J. M. Baribeau, Nature (London) 378, 258 (1995).

${ }^{3}$ K. D. Hirschmann, L. Tsybeskov, S. P. Duttagupta, and P. M. Fauchet, Nature (London) 384, 338 (1996).

${ }^{4}$ D. Leong, M. Harry, K. J. Reeson, and K. P. Homewood, Nature (London) 387, 386 (1997).

${ }^{5}$ W. Theib, Surf. Sci. Rep. 29, 91 (1997).

${ }^{6}$ R. Collins and M. Tischler, Phys. Today 50, 24 (1997).

${ }^{7}$ I. W. Lyo and Ph. Avouris, Science 253, 173 (1991).

${ }^{8}$ K. Prabhakaran and T. Ogino, Surf. Sci. 387, L1068 (1997).

${ }^{9}$ K. Prabhakaran, T. Nishioka, K. Sumitomo, Y. Kobayashi, and T. Ogino, Appl. Surf. Sci. 75, 341 (1994).

${ }^{10}$ K. Prabhakaran, T. Nishioka, K. Sumitomo, Y. Kobayashi, and T. Ogino, Appl. Phys. Lett. 62, 864 (1993); ibid. 64, 1839 (1994).

${ }^{11}$ CRC Handbook of Chemistry and Physics (CRC Boca Raton, FL, 1989).

${ }^{12}$ H.-J. Grossman and E. F. Schubert, Crit. Rev. Solid State Mater. Sci. 18, 1 (1993)

${ }^{13}$ G. Guha, M. D. Pace, D. N. Dunn, and I. L. Singer, Appl. Phys. Lett. 70, 1207 (1997).

${ }^{14}$ V. Craciun, C. B. Leborgne, E. J. Nicholls, and I. W. Boyd, Appl. Phys. Lett. 69, 1506 (1996).

${ }^{15}$ M. Zacharias and P. M. Fauchet, Appl. Phys. Lett. 71, 380 (1995).

${ }^{16}$ A. J. Kenyon, P. F. Trwoga, C. W. Pitt, and G. Rehm, J. Appl. Phys. 79, 9291 (1996).

${ }^{17}$ P. Mutti, G. Ghislotti, S. Bertoni, L. Bonoldi, G. F. Cerofolini, L. Meda, E. Grilli, and M. Guzzi, Appl. Phys. Lett. 66, 851 (1995).

${ }^{18}$ K. S. Min, K. V. Shcheglov, C. M. Yang, H. Atwater, M. L. Brongersma, and A. Polman, Appl. Phys. Lett. 68, 2511 (1996).

${ }^{19}$ L. Skuja, J. Non-Cryst. Solids 167, 229 (1994).

${ }^{20}$ H. R. Philipp, J. Phys. Chem. Solids 32, 1935 (1971).

${ }^{21}$ A. Anedda, G. Bongiovanni, M. Cannas, F. Congui, A. Mura, and M. Martin, J. Appl. Phys. 74, 6993 (1993).

${ }^{22}$ H. Tamura, M. Ruckschloss, T. Wirchem, and S. Veprek, Appl. Phys. Lett. 65, 1537 (1994).

to AIP license or copyright, see http://apl.aip.org/apl/copyright.jsp 